\title{
Fermion family recurrences in the Dyson-Schwinger formalism
}

\author{
Felipe J. Llanes-Estrada ${ }^{1, a}$, Tim Van Cauteren ${ }^{1,2}$, Ángel P. Martín ${ }^{1}$ \\ ${ }^{1}$ Depto. Física Teórica I, Universidad Complutense de Madrid, Avda. Complutense s/n, 28040 Madrid, Spain \\ 2 Dept. Subatomic and Radiation Physics, Proeftuinstraat 86, Ghent University, 9000 Ghent, Belgium
}

Received: 31 August 2006 / Revised version: 9 May 2007 /

Published online: 13 July 2007 - (C) Springer-Verlag / Società Italiana di Fisica 2007

\begin{abstract}
We study the multiple solutions of the truncated propagator Dyson-Schwinger equation for a simple fermion theory with Yukawa coupling to a scalar field. Upon increasing the coupling constant $g$, other parameters being fixed, more than one non-perturbative solution breaking chiral symmetry becomes possible and we find these numerically. These "recurrences" appear as a mechanism to generate different fermion generations as quanta of the same fundamental field in an interacting field theory, without assuming any composite structure. The number of recurrences or flavors is reduced to the question of the value of the Yukawa coupling, and it has no special profound significance in the standard model. The resulting mass function can have one or more nodes and the measurement that potentially detects them can be thought of as a collider-based test of the virtual dispersion relation $E=\sqrt{p^{2}+M\left(p^{2}\right)^{2}}$ for the charged lepton member of each family. This requires the three independent measurements of the charged lepton's energy, threemomentum and off-shellness. We illustrate how this can be achieved for the (more difficult) case of the tau lepton.
\end{abstract}

PACS. 12.15.Ff; 11.30.Rd

\section{Introduction}

Why nature has laid down exactly three fermion families in the same representation of the standard model's gauge groups with universal couplings to the gauge bosons remains to be explained. Many ideas have been presented in the literature, and we quote some to exemplify the far-reaching implications of any experimental progress in the first question of what has been called the "fermion problem".

"Democratic approaches" [1] are in general based on the idea of equal fermion-to-Higgs boson Yukawa couplings. One then needs mechanisms to generate specific lepton or quark mass patterns; see for example [2]. Let us also recall the classic work of Froggat and Nielsen [3], which introduces the concept of "horizontal flavor" symmetry (nowadays often called interfamily symmetry) by which the three families are degenerate or quasi-degenerate at some high scale beyond present experimental reach, due to unknown symmetry. At lower energies, dynamical (renormalization group) effects amplify any small symmetry breaking term causing the ratios between masses that we see in current accelerators. In essence, the idea is that each of the elementary particle types, for example the charged leptons $(e, \mu, \tau)$, provide a representation of the

\footnotetext{
a e-mail: fllanes@fis.ucm.es
}

posited interfamily symmetry. Since the symmetry is broken at the $\mathrm{TeV}$ scale and below, it reveals that it requires theoretical extrapolation from current experimental data. The same authors have followed this up with ideas based on anti-grand unification [4].

An alternative line of research assumes that the known elementary particles are really composite objects of more fundamental "preons" [5]. To date no experiment has revealed any composite structure beyond the usual layers of quantum pairs involved in radiative corrections around seemingly point-like sources. One can argue that a new strong interaction binds preons, and this might conceivably be revealed in future experimental efforts.

Further, dynamical breaking of chiral symmetry as a genuine quantum effect is well known in strong interaction physics $[6,7]$ and has also been invoked in flavor physics as an alternative to the tree-level Higgs mechanism, for example by Brauner and Hosek [8].

However, no one seems to have paid attention to the fact that the Dyson-Schwinger equations do provide one with several solutions depending on the strength of the coupling. In this work we call attention to this point and its potential relevance for the fermion problem. Each of the three fermions corresponds in this hypothesis to a quantum over a different vacuum (but in the covariant formalism one pursues the study of correlation functions, here propagators, and sidesteps the issue of the vacuum wave functional). Far from attempting to reach a complete theory, 
we will show the general features of the mechanism within a simple model of a fermion field coupled to a scalar boson through Yukawa coupling.

From this prospective, different flavored fermions, one corresponding to each family, are quanta of the same field, so the Lagrangian can be more economically written. Each fermion is an elementary one-particle excitation on top of a vacuum that is a local minimum of the Hamiltonian. The excitations over the ground state vacuum provide the lightest fermion family. Finally, at fixed coupling $g$, the DysonSchwinger equation has a finite number of solutions, so a finite number of families arise. The question of why there are three families becomes only a question of the value of $g$, the interaction coupling, and may have no special significance.

The equivalent of the propagator Dyson-Schwinger equation in a non-covariant framework is the well studied mass gap equation of potential models of QCD. The equation has been numerically solved for the harmonic oscillator potential $[9,10]$, the linear potential [11], and the linear plus Coulomb potential $[12,13]$. Bicudo, Ribeiro and Nefediev have systematically studied the excited solutions of this equation [15]. Our results seem compatible with this prior work.

\section{Dyson-Schwinger equation and its numerical solution}

We examine a simple Yukawa theory for one fermion field coupled to a real scalar field with Lagrangian density in Euclidean space

$$
\mathcal{L}=\bar{\Psi}\left(\mathrm{i} \not p-m_{\Psi}\right) \Psi+g \bar{\Psi} \Psi \phi+\frac{1}{2} \phi\left(\partial_{\mu} \partial^{\mu}-m_{\phi}^{2}\right) \phi .
$$

The Dyson-Schwinger equation for the fermion propagator in this theory is represented in Fig. 1 in the rainbow approximation (neglecting the vertex dressing).

To examine the generic features of dynamical chiral symmetry breaking we can further ignore the running of the wavefunction renormalization and set $Z\left(p^{2}\right)=1$, a constant. This leaves one scalar equation for the fermion mass function, which reads

$$
M\left(p^{2}\right)=m_{\Psi} Z_{2}+\int_{0}^{\infty} \mathrm{d} q F(q, p) \frac{M\left(q^{2}\right)}{q^{2}+M^{2}\left(q^{2}\right)} .
$$

In the chiral limit, $m_{\Psi} \rightarrow 0$, this equation becomes homogeneous and accepts a chiral symmetry preserving solution $M=0$ that continuously deforms into a soft-running mass form when $m_{\Psi}$ is not zero. But for strong enough kernels $F$ it also admits other solutions, which break chiral symmetry.

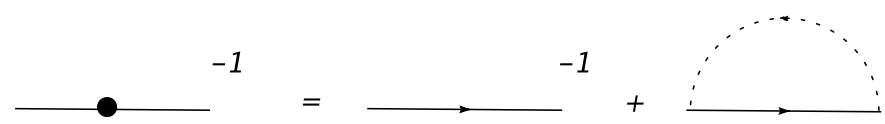

Fig. 1. Dyson-Schwinger equation for the fermion propagator in Yukawa theory, in the rainbow approximation
The kernel in this simple Yukawa theory is

$$
F(q, p)=\frac{g^{2} q^{3}}{4 \pi^{3}} \int_{-1}^{1} \sqrt{1-y^{2}} \mathrm{~d} y \frac{1}{(q-p)^{2}+m_{\phi}^{2}},
$$

with $y$ the cosine of the polar angle in Euclidean fourdimensional space from $p$ to $q$.

To obtain excited solutions for $M$ as a function of $p^{2}$ we apply an iterative linear method with different initial guesses with or without nodes for $M\left(p^{2}\right)$. This method proceeds by examining linear deviations of the exact solution

$$
M\left(p^{2}\right)=M_{0}\left(p^{2}\right)+\varepsilon\left(p^{2}\right),
$$

which yield

$$
\begin{aligned}
& \varepsilon\left(p^{2}\right)-\int_{0}^{\infty} \mathrm{d} q F(q, p) \varepsilon\left(q^{2}\right)\left(\frac{q^{2}-M^{2}\left(q^{2}\right)}{\left(q^{2}+M^{2}\left(q^{2}\right)\right)^{2}}\right) \\
& =m_{\Psi} Z_{2}-M_{0}\left(p^{2}\right)+\int_{0}^{\infty} \mathrm{d} q F(q, p) \frac{M_{0}\left(q^{2}\right)}{q^{2}+M_{0}^{2}\left(q^{2}\right)} .
\end{aligned}
$$

This can in turn be discretized (simply discretizing $p$ ) and cast as a linear system:

$$
A_{i j} \varepsilon_{j}=(R H S)_{i},
$$

and this can be solved with standard numerical linear algebra tools. The kernels require only the calculation of twodimensional integrals that are also standard fare in modern computers.

By naive dimensional analysis one sees that the integral needs UV regularization. We accomplish this by a simple cut-off method. Then the parameters should be chosen to run with the cut-off $g(\Lambda)$ and $m_{\psi}(\Lambda)$ to ensure that the mass functions turn largely independent of $\Lambda$. In our results we display the dependence of the solution with $g$ at fixed $\Lambda$. The boson mass $m_{\phi}$ is chosen to be 1 and sets the unit of the theory.

\section{Numerical results and discussion}

In this section we present the chiral symmetry breaking conventional and new excited state solutions. By running our computer program incrementing $g$ we meet critical $g$ values $g_{0}, g_{1}, \ldots, g_{n}$ above which there are exactly $n+1$ solutions, the last with $n$ nodes, that we find numerically. For example, the first solution appears for a critical value of $g_{0} \simeq 6.8$ (of course this number runs with the cut-off).

We show in Fig. 2 the three solutions obtained with $g=$ 14 and $\Lambda=200$.

Next in Fig. 3 we plot the nodeless solution at fixed cutoff incrementing $g$ sequentially to show the dependence of the mass function on the coupling.

Only the nodeless solution has been widely used in the past literature for its applications in hadron physics. The others are sometimes rejected $[16,17]$ on the basis of a "wrong" UV asymptotic behavior, arguing that they do not match with conventional mass running in perturbation 


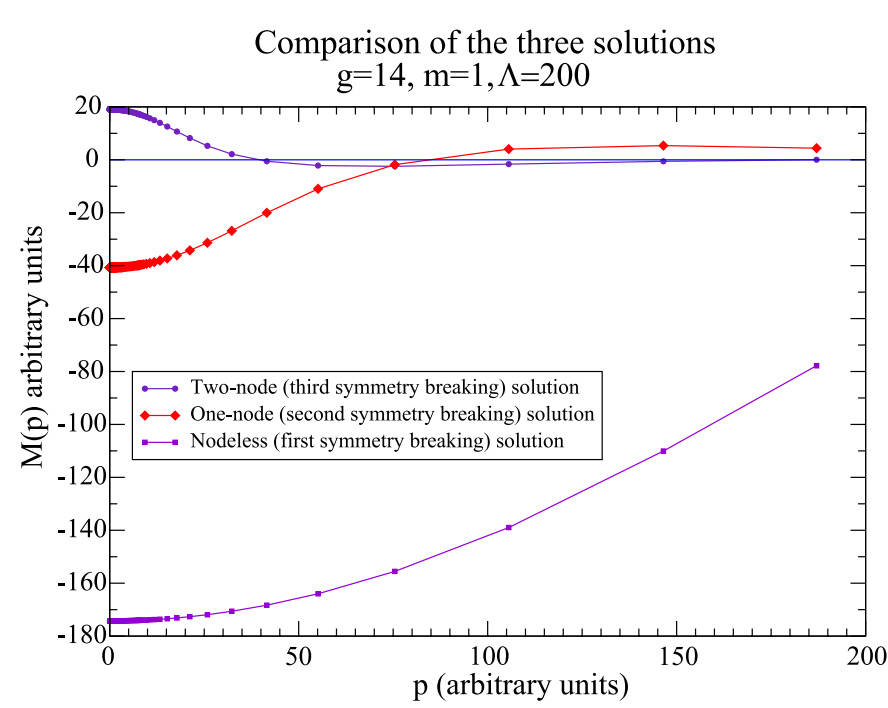

Fig. 2. We plot together the three solutions to the DysonSchwinger equation for a fermion-scalar Yukawa coupling $g=14$, cutoff $\Lambda=200$, and the scale set by the scalar mass being fixed to $m_{\phi}=1$. Note the equation for $M$ in the chiral limit (zero current fermion mass) cannot distinguish $M$ from $-M$ so the global sign of any one solution in this graph is irrelevant and for display purposes only. In particular all $\mathrm{M}(0)$ can be taken as positive

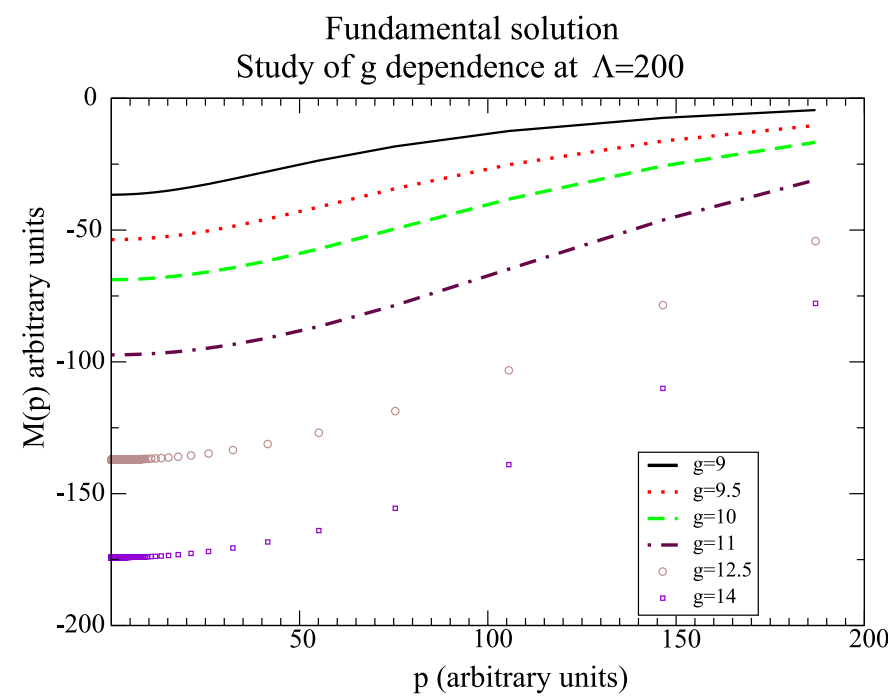

Fig. 3. Nodeless solution for increasing values of the coupling constant $\left(m_{\phi}=1\right.$ and $\Lambda=200$ are held fixed). Note how $M(0)$ grows steadily with $g$ (the sign of $M$ is irrelevant)

theory, and artificially imposing a boundary condition that discards all but one solution.

We point out here that this mismatch poses no problem. One should interpret these solutions as the one-particle excitations above different extrema of the Hamiltonian or vacuum replicae. Therefore they cannot all be matched to the same perturbation theory around one particular vacuum.

If one insists on viewing them from the one vacuum that connects smoothly to perturbation theory, then they

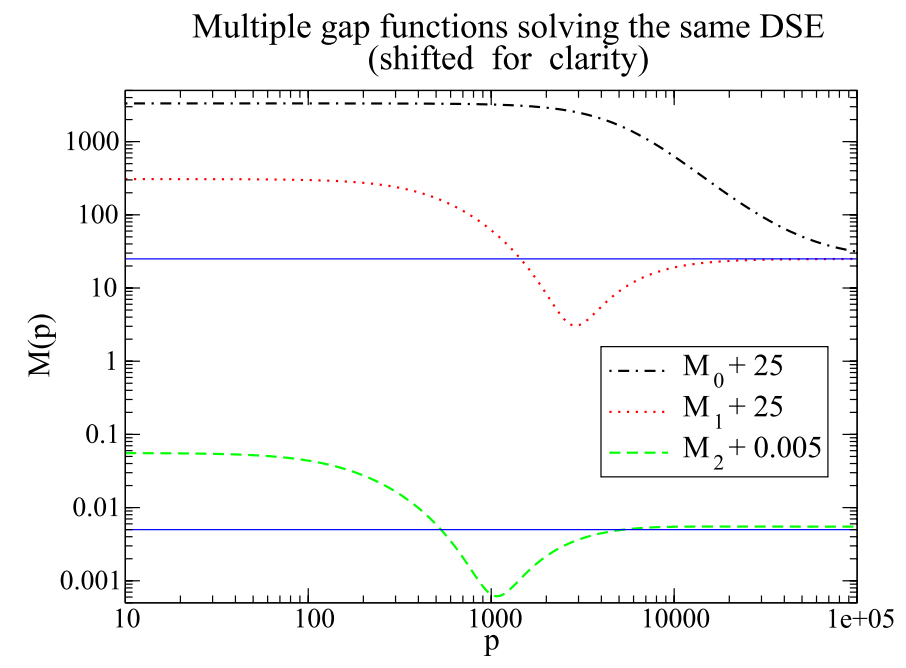

Fig. 4. Three solutions to the gap equation in Yukawa theory obtained with $m_{\phi}=100, \Lambda=10^{4}, m_{\psi}=5 \times 10^{-4}, g=50$, confirming that the nodeless solution is higher in mass, and that a sizeable hierarchy of masses is possible. To represent them in a log-log plot, $M_{1}$ and $M_{2}$ being negative in part of their domain, the solutions have been shifted by a constant amount to positive values

have to be written down as complicated collective states in terms of the one-particle solutions over this vacuum.

In the particular case of the Yukawa theory, the angular integral in (3) can be performed analytically [14], but we prefer keeping a two-dimensional integral thinking of future work with more complicated model Lagrangians.

In agreement with the findings of [15], we see that the higher excited vacua in this covariant Euclidean formulation present zeroes in the mass function. A version of the Sturm-Liouville theorem must be at work (this is not surprising since the Hamiltonian is hermitian).

Also note that the values of $M(0)$ generated in this covariant Yukawa theory show quite some hierarchy. In Fig. 2 one can see that they are well-spaced. Therefore this fieldtheory mechanism might conceivably be at work in the fermion flavor problem. However, $M(0)$ seems to be larger for the nodeless solution than for the excited ones (this puzzling property also appears in the equal-time approach of [15], and the extent of its model dependence needs to be further investigated). To confirm this point, we choose a different set of parameters and implementation of the computer code. With $m_{\phi}=100$, a cut-off $\Lambda=10^{4}$ implemented as a Gaussian fall-off of the integration measure (as opposed to terminating the grid), and $g=50$, with a small bare fermion mass $m_{\psi}=5 \times 10^{-4}$, one obtains the three solutions depicted in Fig. 4. This graph confirms that the nodeless solution is higher in mass, and that it is possible to generate sizeable mass hierarchies between the solutions. We use this run to also illustrate the dependence of $M_{2}$ on the cut-off, displayed in Fig. 5. As can be seen, the excited solution does not decrease monotonically as $g \rightarrow 0$; it rather seems to become a singular solution at the critical coupling. This technicality is however probably irrelevant for physical applications. 


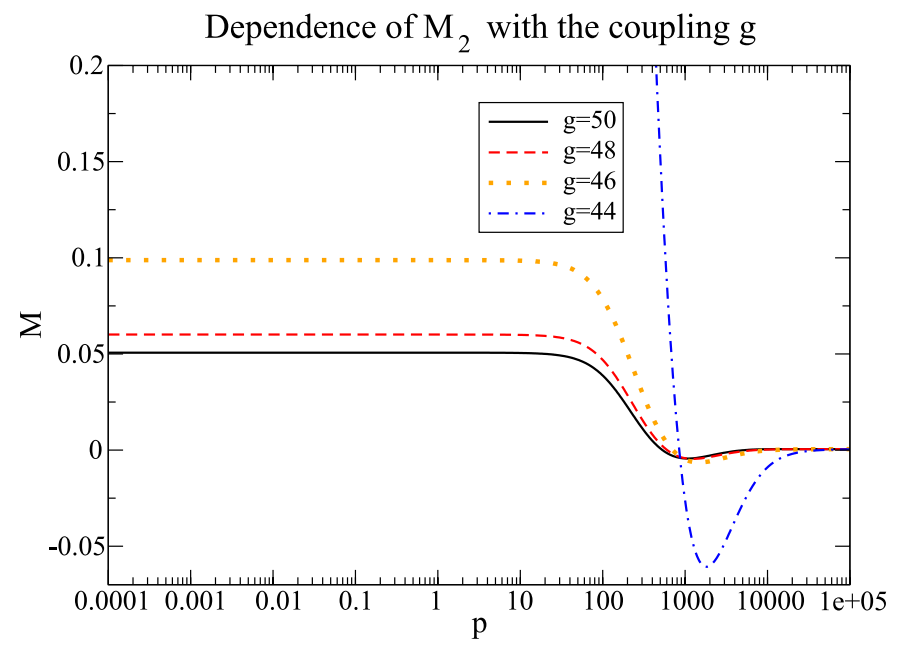

Fig. 5. Dependence of $M_{2}$ with the coupling constant at fixed $m_{\phi}=100, \Lambda=10^{4}, m_{\psi}=5 \times 10^{-4}$. Upon comparing with Fig. 3 we see that the qualitative behavior when the solution approaches the critical coupling and is about to cease existing, here $g=43$, is quite different for the ground state and excited solutions

In hadron physics, the strength of the interaction being fixed by extensive phenomenology, there would be no freedom to vary $g$. In the physically relevant cases studied in [15], excited solutions exist at physical values of the coupling. In [18] Ribeiro and Nefediev have further extended their original results to include bound states (mesons) constructed with the replicated quasiparticles. In particular there are Goldstone bosons over the replicated vacuum, that are of course not massless pions but would appear as excited pion states (separated from the ground state pion by the mass gap between the two vacua). Whether any conventional mesons accept a more convenient description as replicated mesons instead of excited mesons over the standard vacuum remains an open question.

Working in Euclidean space as we do provides fastly convergent integrals. This technicality may be avoided by employing a Lehmann representation [19] that allows for a direct solution in Minkowski space with similar results.

\section{Experimental signature}

In a free field theory, a field quantum of mass $m$ satisfies the equation

$$
E=\sqrt{\mathbf{p}^{2}+m^{2}}
$$

which causes a pole in the free propagator,

$$
S=\frac{\mathrm{i}}{p^{2}-m^{2}} .
$$

In an interacting field theory this pole will be shifted due to renormalization from its bare to its physical position. The dependence on the renormalization scale can

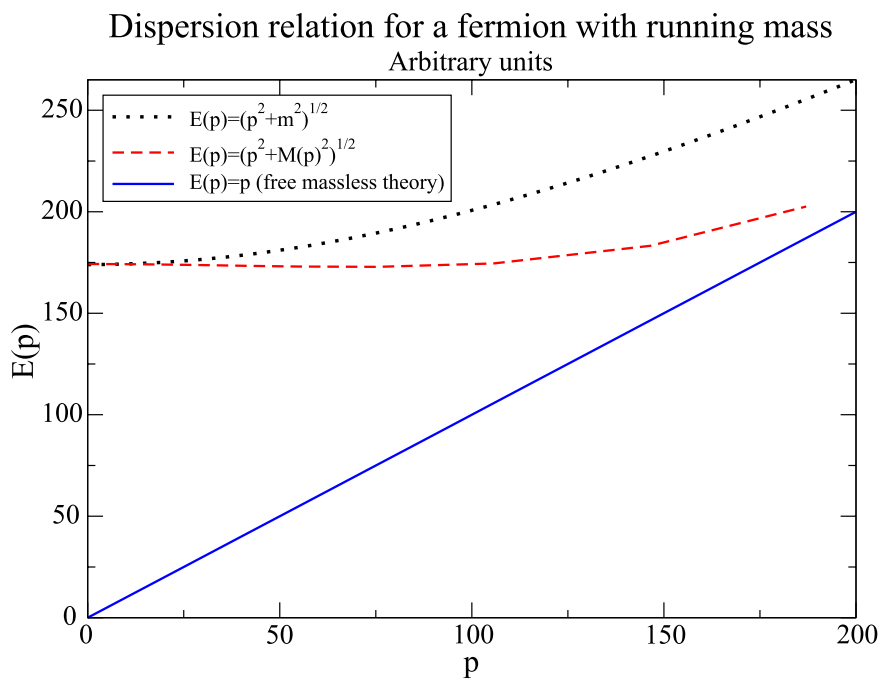

Fig. 6. The virtual particle dispersion relation at fixed cutoff scale for the nodeless $M\left(p^{2}\right)$ solution. Note that the interacting theory falls below the usual Yukawa dispersion relation because the mass function drops with $p$. This is not very different from standard perturbative running with $p$ except that the effect is much larger in the case of dynamical chiral symmetry breaking

be traded by a dependence on the particle Euclidean four-momentum, so that

$$
S=\frac{\mathrm{i} Z\left(p^{2}\right)}{p^{2}-M\left(p^{2}\right)^{2}}
$$

yielding the transcendental equation for the pole position

$$
E=\sqrt{\mathbf{p}^{2}+M\left(E^{2}-\mathbf{p}^{2}\right)^{2}} .
$$

Another way of visualizing the function $M\left(p^{2}\right)$ of the interacting theory is to trade $p^{2}$ by the space-like part $\mathbf{p}^{2}$ as argument of $M$, generating therefore a non-trivial dispersion relation for virtual particles, and the zeroes of the mass function $M$ appear as points where $E=|\mathbf{p}|$ that would otherwise only be reached asymptotically at large p (see Fig. 7). Tests for a non-trivial (real particle) dispersion relation have been proposed, and some have been carried out in the search for violations of Lorentz invariance [20]. However, these are very indirect and usually are performed at low energy, so an accelerator-based test of the (virtual particle) dispersion relation by separately measuring $E, \mathbf{p}$ and the off-shellness is preferable. In our case, real particles still provide representations of the Poincaré group and have constant, physical mass. However, virtual particles off their mass shell will display the running mass function, and this can be captured by analyzing the amplitudes of physical processes in perturbation theory.

The corresponding dispersion relations are plotted in Figs. 6 and 7.

Lacking a real theory there are two possibilities we cannot discern at present. Either all three families of fermions arise as solutions spontaneously breaking chiral symmetry, in which case two of them should already have a zero in their mass function, or the first generation reflects the trivial solution only slightly modifying the bare mass, and the 


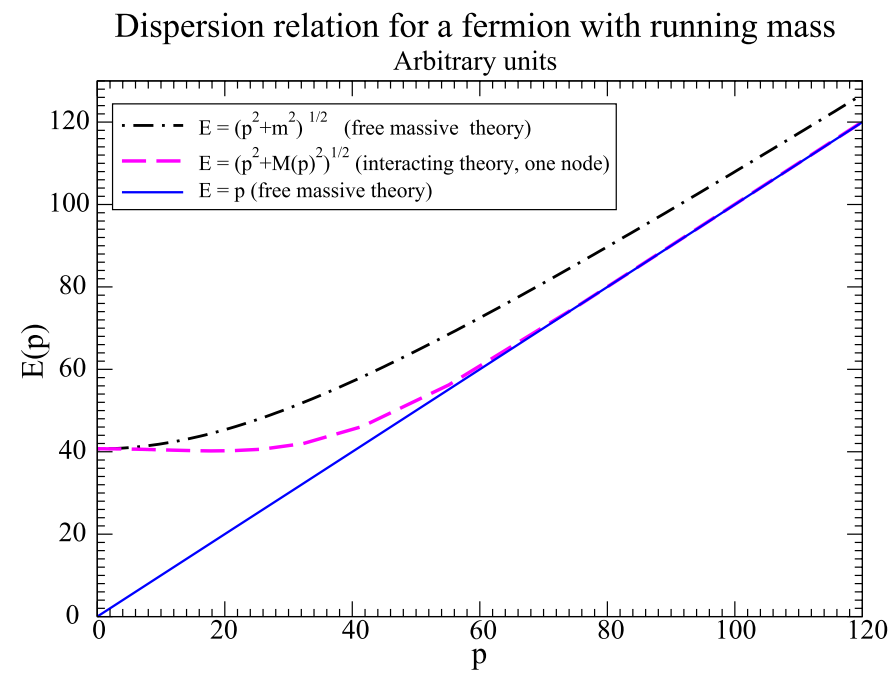

Fig. 7. The virtual dispersion relation at fixed renormalization scale for the solution with two nodes (we find similar result for one node). As the running mass actually has a zero, the dispersive curve touches the massless limit that would otherwise be reached only asymptotically

second and third generation solutions with dynamically broken chiral symmetry, in which case only one of them would present a node. In either case a zero is present in the mass function of one of the fermions.

An interesting experiment is therefore a measurement of $\mathbf{p}, E$ and the off-shellness $\Delta^{2}=p^{2}-M^{2}\left(p^{2}\right)$ of a $\tau$, at various momenta $\mathbf{p}$. The $B$ factories have accumulated large $\tau$ samples [21], which are under analysis and can be employed for this purpose.

The process one may investigate is depicted in Fig. 10 in the appendix below. An electron-positron pair collides and annihilates into a $\tau^{-*} \tau^{+}$-pair, where the $\tau^{-*}$ is off its mass shell. Its four-momentum can be inferred from the centerof-momentum energy of the incoming $e^{-} e^{+}$-pair and by reconstructing the energy and momentum of the on-shell $\tau^{+}$from its decay products, as we treat in detail shortly. Finally, one needs the off-shellness, but this can be obtained from the number of counts with given $E,|\mathbf{p}|$. This is obvious since the cross section for a specific process, where the off-shell $\tau^{-}$decays into for example $l^{-} \bar{\nu}_{l} \nu_{\tau}$, depends on the off-shellness $\Delta^{2}$ of the $\tau^{-}$(see also the appendix).

Let us now give details for the possible analysis.

1. In the center of mass frame of an $e^{-} e^{+}$collision one can identify two back-to-back hadron jets, tagging the flavor by demanding that one of them kinematically reconstructs an on-shell $\tau$. This is rendered difficult by the undetected neutrino, which we assume to be the only unreconstructed track on the left side of the event.

2. Complete reconstruction of the energy and momentum on the left side of the event (see Fig. 8) is possible with a vertex detector (giving the direction of motion of the on-shell $\tau$ ). The total energy is taken from collider calibration and matched to the energy of the visible tracks. Balancing energy provides the energy of the missing neutrino, and automatically the momentum of the left

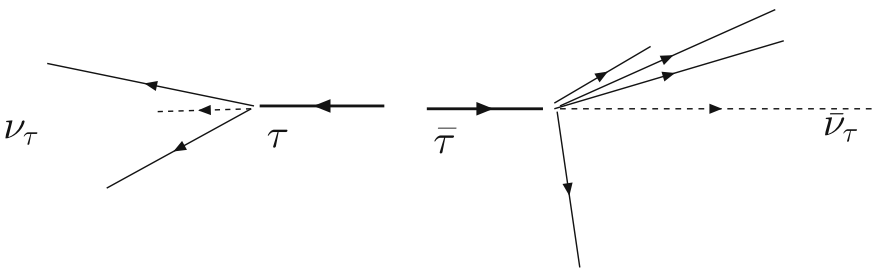

Fig. 8. In center of mass frame production where $\mathbf{p}_{\tau}+\mathbf{p}_{\bar{\tau}}=\mathbf{0}$ one can fully reconstruct the decay of both $\tau$ leptons if only the neutrinos escape undetected. The analysis steps are described in the text

side, which can be tested against the hypothesis of a physical $\tau$ being produced.

3. Next, one examines the right side of the reaction, where the secondary and primary vertex seem to coincide (as appropriate for a virtual, off-shell $\tau$ decaying rapidly). The total three-momentum is known a priori. One balances momentum to obtain the momentum taken by the undetected $\nu_{\tau}$, and one obtains the total energy. Now, it is not necessarily true that $E^{2}-\mathbf{p}^{2}=m_{\tau}^{2}$.

4. The number of counts with given $E, \mathbf{p}$ on the right side is normalized to a cross section in terms of the offshellness $\sigma\left(\Delta^{2}\right)$ and used to obtain $M$ from the value of the intermediate propagator in perturbation theory.

An alternative possibility is to carry out the measurement on the expected $O(20-30) \tau$ events at the OPERA experiment [22] in Gran Sasso National Laboratory. Given the momentum of the neutrinos from the CNGS beam one can attempt full kinematical reconstruction of $(E, \mathbf{p})$. However, here the difficulty resides in the off-shellness, since a small value will be forced for the identification of the $\tau$.

The experimental test for the muon and the electron is even simpler, for example, through Compton and reverse Compton scattering, where the fermion is off-shell in the intermediate state. For quarks one needs to take into account that they always appear in bound states, and therefore the mass function is always under an integral sign, and reconstruction from the experimental data is difficult. However, a particularly simple case is

$$
e^{-} e^{+} \rightarrow \gamma^{*} \rightarrow s \bar{s},
$$

where the strangeness is completely tagged by counting all hyperons in both jets.

In principle, the same analysis as carried out here could also be undertaken for Majorana particles, but this is out of the scope of this preliminary work.

\section{Conclusions}

We have called attention to an interesting feature of field theory, namely the possibility of having several solutions of the one-particle Dyson-Schwinger equations for a broad class of theories. The excited solutions appear upon increasing the coupling constant sufficiently, breaking chiral symmetry. 
The spectrum of states on top of each of the vacua is called "replica" or "recurrence" and provides a mechanism that might be interesting for the theory of flavor. The number of recurrences arises from no special symmetry, since non-linear equations can perfectly have a finite number of solutions (again depending on the value of the coupling; this can be chosen to be three).

These solutions have been shown in the past in a Hamiltonian framework [15], and we have minimally extended the results of these authors to show that the covariant formulation allows for similar phenomena. The excited solutions have mass functions that present zeroes and this is reflected in the virtual dispersion relation (a feature of an interacting field theory that should not be confused with breaking of Lorentz invariance).

Whether these solutions will be relevant to the fermion family problem can be tested at $B$ factories employing their large $\tau$ samples directly produced.

At this point it is a meaningless exercise to attempt to obtain the fermion masses in this scheme. Three data points $M_{i}(0)$ should be used to fit three parameters, the current fermion mass (that we have here set to zero), the boson mass, and $g$ at a given cut-off, so the current predictive power is null. However, we find this field-theory mechanism still to be worth of attention given the ease with which fermion coupling universality can be incorporated by having three recurrences of the same field.

Testing the off-shell dispersion relation should allow one to immediately discard the scenario, so we believe we have presented a valid physical hypothesis. Of course, the success of radiative corrections in the standard model makes one wish for a measurement at the highest possible energy, maybe at a future linear collider.

Acknowledgements. This work has been performed in the framework of the research projects FPA 2004-02602, 200502327, PR27/05-13955-BSCH (Spain) and is part of the Masters thesis of Mr. Páramo Martín presented to the faculty of U. Complutense). TVC is a postdoctoral fellow for the Fund for Scientific Research - Flanders and acknowledges the support of the "Programa de Investigadores Extranjeros en la UCM Grupo Santander".

\section{Appendix : $\tau$-pair production in $e^{+} e^{-}$ collisions}

\section{A.1 On-shell cross section}

We look at the $e^{+} e^{-} \rightarrow \tau^{+} \tau^{-}$process depicted in Fig. 9, where all ingoing and outgoing particles are on their mass shell. Using the conventions of [23], the amplitude for the depicted process is

$$
\begin{aligned}
-\mathrm{i} \mathcal{M}= & \bar{u}_{\eta^{-}}^{\tau^{-}}\left(p^{-}\right)\left(-\mathrm{i} e \gamma^{\nu}\right) v_{\eta^{+}}^{\tau^{+}}\left(p^{+}\right)\left(\frac{-\mathrm{i} g_{\mu \nu}}{Q^{2}}\right) \\
& \times \bar{v}_{\xi^{+}}^{e^{+}}\left(k^{+}\right)\left(\mathrm{i} e \gamma^{\mu}\right) u_{\xi^{-}}^{e^{-}}\left(k^{-}\right) .
\end{aligned}
$$

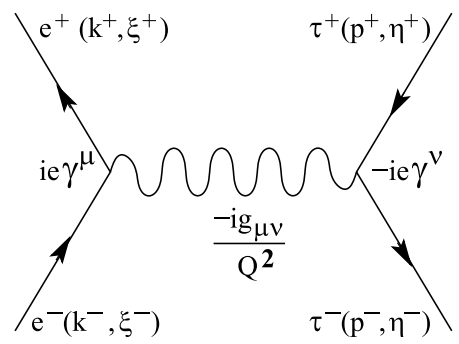

For the unpolarized cross section, one needs the squared amplitude averaged over initial and summed over final spins:

$$
\begin{aligned}
& \bar{\sum}|\mathcal{M}|^{2}=\frac{1}{4} \frac{e^{4}}{s^{2}} \sum_{\text {all spins }} \bar{u}_{\eta^{-}}^{\tau^{-}}\left(p^{-}\right) \gamma_{\mu_{\eta^{-}} \eta^{+}} v_{\eta^{+}}^{\tau^{+}}\left(p^{+}\right) \\
& \times \bar{v}_{\xi^{+}}^{e^{+}}\left(k^{+}\right) \gamma_{\xi^{+} \xi^{-}} u_{\xi^{-}}^{e^{-}}\left(k^{-}\right) \bar{u}_{\xi^{-}}^{e^{-}}\left(k^{-}\right) \gamma_{\xi^{\prime}}^{\nu} \xi^{\prime+} v^{e^{+}}{ }_{\xi^{\prime}}\left(k^{+}\right) \\
& \times \bar{v}_{\eta^{\prime}}^{\tau^{+}}\left(p^{+}\right) \gamma_{\nu{ }_{\eta^{\prime}}{ }_{\eta^{\prime}}} u_{\eta^{\prime}}^{\tau^{-}}\left(p^{-}\right),
\end{aligned}
$$

where the spinor indices are explicitly shown. For $e^{+} e^{-}$ annihilation, one has $Q^{2}=s$. One can now rearrange the factors in (A.2). Making use of the relations

$$
\begin{aligned}
u_{\xi}^{l}(p) \bar{u}_{\xi^{\prime}}^{l}(p) & =\left(\not p+m_{l}\right)_{\xi \xi^{\prime}}, \\
v_{\xi}^{l}(p) \bar{v}_{\xi^{\prime}}^{l}(p) & =\left(\not p-m_{l}\right)_{\xi \xi^{\prime}},
\end{aligned}
$$

one can write (A.2) as a product of traces:

$$
\begin{aligned}
\bar{\sum}|\mathcal{M}|^{2}= & \frac{1}{4} \frac{e^{4}}{s^{2}} \operatorname{Tr}\left[\left(\not k^{-}+m_{e}\right) \gamma^{\nu}\left(k^{+}-m_{e}\right) \gamma^{\mu}\right] \\
& \times \operatorname{Tr}\left[\left(p^{-}+m_{\tau}\right) \gamma_{\mu}\left(p^{+}-m_{\tau}\right) \gamma_{\nu}\right] .
\end{aligned}
$$

Neglecting the electron mass, this results in

$$
\begin{aligned}
\bar{\sum}|\mathcal{M}|^{2}=\frac{8 e^{4}}{s^{2}}\left(k^{-} \cdot p^{-} k^{+} \cdot p^{+}\right. & +k^{-} \cdot p^{+} k^{+} \cdot p^{-} \\
& \left.+m_{\tau}^{2} k^{-} \cdot k^{+}\right) .
\end{aligned}
$$

This can be written in terms of the Mandelstam variables,

$$
\begin{aligned}
s & =\left(k^{-}-k^{+}\right)^{2}=\left(p^{+}-p^{-}\right)^{2}=Q^{2}, \\
t & =\left(k^{-}-p^{-}\right)^{2}=\left(p^{+}-k^{+}\right)^{2}, \\
u & =\left(k^{-}-p^{+}\right)^{2}=\left(p^{-}-k^{+}\right)^{2},
\end{aligned}
$$

as follows:

$$
\bar{\sum}|\mathcal{M}|^{2}=\frac{2 e^{4}}{s^{2}}\left(t^{2}+u^{2}+4 m_{\tau}^{2} s-2 m_{\tau}^{4}\right)
$$

where at high energies the mass terms become negligible. In the center-of-momentum (CoM) frame, the amplitude is

$$
\begin{aligned}
\bar{\sum}|\mathcal{M}|^{2}=\frac{e^{4}}{s^{2}}[ & s^{2}\left(1+\cos ^{2}\left(\theta_{\tau}\right)\right) \\
& \left.+4 m_{\tau}^{2} s\left(1-\cos ^{2}\left(\theta_{\tau}\right)\right)\right],
\end{aligned}
$$


with $\theta_{\tau}$ the CoM angle between incoming electron $e^{-}$and outgoing $\tau^{-}$.

The differential cross section in the CoM frame is then given by

$$
\begin{aligned}
\left.\frac{\mathrm{d} \sigma}{\mathrm{d} \Omega}\right|_{\mathrm{CoM}}= & \frac{\alpha^{2}}{4 s^{2}} \sqrt{1-\frac{4 m_{\tau}^{2}}{s}} \\
& \times\left[s\left(1+\cos ^{2}\left(\theta_{\tau}\right)\right)+4 m_{\tau}^{2} \sin ^{2}\left(\theta_{\tau}\right)\right],
\end{aligned}
$$

with $\alpha=e^{2} / 4 \pi$. The total cross section is

$$
\sigma_{\text {tot. }}^{\mathrm{CoM}}=\frac{4 \pi \alpha^{2}}{3 s} \sqrt{1-\frac{4 m_{\tau}^{2}}{s}}\left(1+\frac{2 m_{\tau}^{2}}{s}\right) .
$$

\section{A.2 Half off-shell cross section}

In this part of the appendix, we look at the $e^{-} e^{+} \rightarrow \tau^{-} \tau^{+}$ process, where one of the $\tau$-particles is off its mass shell and decays into a lighter lepton $l$ (electron or muon). We discuss the case for an off-shell $\tau^{-}$. The case for an off-shell $\tau^{+}$ is clearly completely analogous. The process is displayed in Fig. 10.

The off-shellness of one of the $\tau$ changes the kinematics. The total energy in the reaction is unevenly divided: $E_{\tau^{+}} \neq E_{\tau^{-}} \neq W_{\mathrm{CoM}} / 2$, where $W_{\mathrm{CoM}}=\sqrt{s}$ is the total energy in the CoM frame. The amplitude for the process depicted in Fig. 10 is

$$
\begin{aligned}
-\mathrm{i} \mathcal{M}= & \bar{u}_{\zeta_{l}}^{l^{-}}\left(p_{l}\right)\left(-\mathrm{i} \frac{g_{W}}{\sqrt{2}} \gamma^{\sigma} \frac{1}{2}\left(1-\gamma^{5}\right)\right) v^{\bar{\nu}_{l}}\left(p_{\bar{\nu}_{l}}\right) \\
& \times(-\mathrm{i}) \frac{\left(g_{\kappa \sigma}-\frac{q_{\kappa}^{W} q_{\sigma}^{W}}{M_{W}^{2}}\right)}{\left(q^{W^{2}}-M_{W}^{2}\right)} \bar{u}^{\nu_{\tau}}\left(p_{\nu_{\tau}}\right) \\
& \times\left(-\mathrm{i} \frac{g_{W}}{\sqrt{2}} \gamma^{\sigma} \frac{1}{2}\left(1-\gamma^{5}\right)\right) \\
& \times \frac{\mathrm{i}}{\left(p^{-}-M_{\tau^{-}}\right)}\left(-\mathrm{i} e \gamma^{\nu}\right) v_{\eta^{+}}^{\tau^{+}}\left(p^{+}\right)\left(\frac{-\mathrm{i} g_{\mu \nu}}{q^{2}}\right) \\
& \times \bar{v}_{\xi^{+}}^{e^{+}}\left(k^{+}\right)\left(\mathrm{i} e \gamma^{\mu}\right) u_{\xi^{-}}^{e^{-}}\left(k^{-}\right) .
\end{aligned}
$$

The propagator of the off-shell $\tau^{-}$will give rise to a dependence of the amplitude on the off-shellness $\Delta^{2}=E_{\tau^{-}}^{2}-\mathbf{p}_{\tau}^{2}-M_{\tau^{-}}^{2}$.

In the limit of infinite mass of the $W$-boson and vanishing electron mass, the unpolarized squared amplitude for the process depicted in Fig. 10 is ${ }^{1}$

$$
\begin{aligned}
& \bar{\sum}|\mathcal{M}|^{2}=\frac{2^{12} \pi^{4} \alpha_{\mathrm{QED}}^{2} \alpha_{\text {weak }}^{2}}{M_{W}^{4} \Delta^{4} q^{4}} p_{\nu_{\tau}} \cdot p_{l} \\
& \times\left[p_{\bar{\nu}_{l}} \cdot p^{-}\left(m_{\tau} M_{\tau^{-}} k^{+} \cdot k^{-}+k^{+} \cdot p^{+} k^{-} \cdot p^{-}+k^{+} \cdot p^{-} k^{-} \cdot p^{+}\right)\right. \\
& \left.\quad-\frac{\Delta^{2}}{2}\left(k^{+} \cdot p_{\bar{\nu}_{l}} k^{-} \cdot p^{+}+k^{+} \cdot p^{+} k^{-} \cdot p_{\bar{\nu}_{l}}\right)\right] .
\end{aligned}
$$

1 The Dirac trace algebra was performed using FORM, version $3.1[24]$.

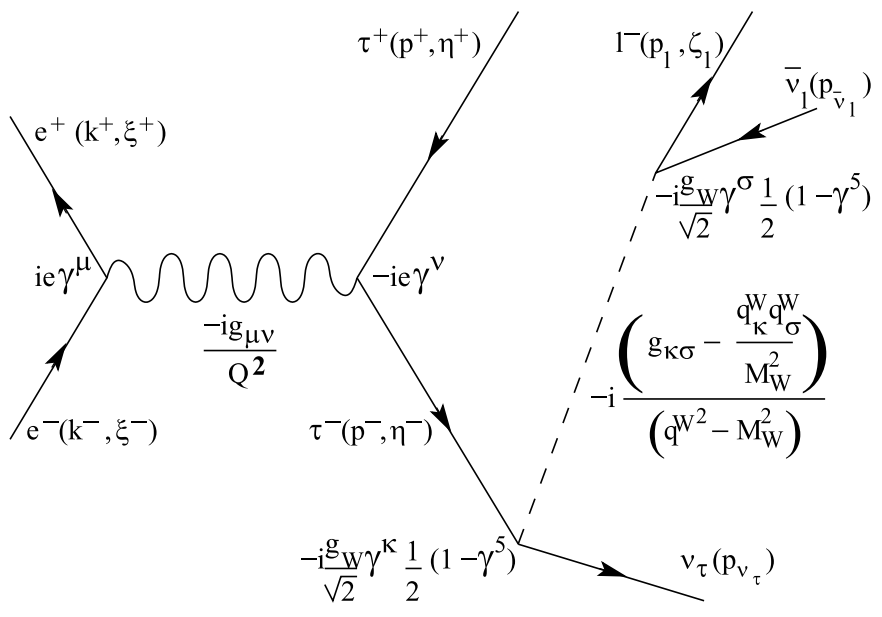

Fig. 10. Half off-shell $\tau$-pair production in $e^{+} e^{-}$collisions. The particles' momenta and spins are indicated, as well as the conventions for the boson propagators and the vertices

The (differential) cross section for this process is proportional to the above squared amplitude, integrated over the three-momenta of the outgoing $\tau$-neutrino and leptonantineutrino. This is a standard computation that can be incorporated in the computer code if needed; yet it is clear that the resulting cross section will depend on the offshellness $\Delta^{2}$ and the virtual $\tau$ mass-energy dispersion relation. In particular, at large off-shellness, the cross section behaves as $1 / \Delta^{2}$ and the constant multiplying this parametric behavior can be fit to the data.

\section{References}

1. H. Fritzsch, Nucl. Phys. Proc. Suppl. 40, 121 (1995) [arXiv:hep-ph/9411419]

2. R. Dermisek, Phys. Rev. D 70, 033007 (2004) [arXiv: hep-ph/0312206]

3. C.D. Froggatt, H.B. Nielsen, Nucl. Phys. B 147, 277 (1979)

4. C.D. Froggatt, H.B. Nielsen, D.J. Smith, Phys. Lett. B 385, 150 (1996) [arXiv:hep-ph/9607250]

5. J.C. Pati, Phys. Rev. D 30, 1144 (1984)

6. C.D. Roberts, arXiv:nucl-th/0301065

7. P. Maris, C.D. Roberts, Int. J. Mod. Phys. E 12, 297 (2003) [arXiv:nucl-th/0301049]

8. T. Brauner, J. Hosek, Phys. Rev. D 72, 045007 (2005) [arXiv:hep-ph/0505231]

9. P.J. de A. Bicudo, J.E.F.T. Ribeiro, Phys. Rev. D 42, 1611 (1990)

10. A. Le Yaouanc, L. Oliver, S. Ono, O. Pene, J.C. Raynal, Phys. Rev. D 31, 137 (1985)

11. S.L. Adler, A.C. Davis, Nucl. Phys. B 244, 469 (1984)

12. A.P. Szczepaniak, E.S. Swanson, Phys. Rev. D 55, 1578 (1997) [arXiv:hep-ph/9609525]

13. F.J. Llanes-Estrada, S.R. Cotanch, Nucl. Phys. A 697, 303 (2002) [arXiv:hep-ph/0101078]

14. F.J. Llanes-Estrada, P.J. de A. Bicudo, Phys. Rev. D 68, 094014 (2003) [arXiv:hep-ph/0306146]

15. P.J. de A. Bicudo, J.E.F.T. Ribeiro, A.V. Nefediev, Phys. Rev. D 65, 085026 (2002) [arXiv:hep-ph/0201173] 
16. A. Kizillersu, private communication

17. A. Kizilersu, T. Sizer, A.G. Williams, Prepared for 5th International Conference on Quark Confinement and the Hadron Spectrum, Gargnano, Brescia, Italy, 10-14 September 2002

18. A.V. Nefediev, J.E.F.T. Ribeiro, Phys. Rev. D 70, 094020 (2004) [arXiv:hep-ph/0409112]

19. V. Sauli, JHEP 0302, 001 (2003) [arXiv:hep-ph/ 0209046
20. D. Mattingly, Living Rev. Relat. 8, 5 (2005) [arXiv:gr-qc/ 0502097]

21. O. Igonkina, arXiv:hep-ex/0606009

22. OPERA Collaboration, arXiv:hep-ex/0611023

23. F. Halzen, A.D. Martin, Quarks \& Leptons: An Introductory Course in Modern Particle Physics, 1st. edn. (John Wiley \& Sons, Inc., New York, 1984)

24. J.A.M. Vermaseren, New features of FORM, math-ph/ 0010025 